\begin{abstract}
Weblinks
www.onkodin.de

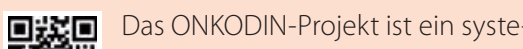
matisches Informationssystem für ג die klinische Onkologie und Hämatologie. Die Seite bietet ein laufend erweiter tes Online-Kompendium sowie Berichte von internationalen Kongressen und Vorträgen. In Kooperation mit dem Kompetenznetz «Akute und chronische Leukämien» und dem «European Leukemia Network» entsteht hier zudem ein Bildatlas der hämatologischen Zytologie. ONKODIN ist eine von Organisationen, Instituten und Firmen inhaltlich unabhängige, kostenfreie Publikationsplattform.
\end{abstract}

www.mamazone.de/brustkrebs

마짐ㅁ mamazone - Frauen und Forschung

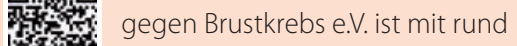

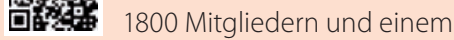
wissenschaftlichen Beirat renommierter Brustkrebsexperten die größte und aktivste Brustkrebs-Patientinnen-Initiative in Deutschland. Sie arbeitet bundesweit. Das Hauptbüro in Augsburg steht als zentrale Anlaufstelle zur Verfügung und koordiniert die 12 regionalen Büros. Die ärztliche Empfehlung dieser Website unterstützt die Betroffenen bei der sorgsamen Nachsorge und stärkt ihre Eigenverantwortung im Umgang mit Brustkrebs.

www.kompetenznetz-kokon.de/home 回裙扣 Das Kompetenznetz Komplemen-

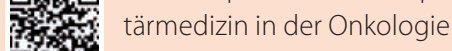
四程 (KOKON) ist ein Verbundforschungs projekt, das die Kenntnis von medizinischem Personal und Patienten im Bereich Komplementärmedizin in der Onkologie verbessern will. Interdisziplinäre Forschungsgruppen erarbeiten und evaluieren Fortbildungskonzepte für Fachleute und Schulungsprogramme für Krebsselbsthilfegruppen. Darüber hinaus bauen sie ein Expertennetzwerk für ärztliche Fachberatung zur Komplementärmedizin in der Onkologie auf. Als Grundlage entstehen Wissensdatenbanken zur Evidenz komplementärmedizinischer Behandlungsverfahren und zu Arzneimittelinteraktionen. Das Kompetenznetz wird bis 2015 von der Deutschen Krebshilfe gefördert und von einem multidisziplinären wissenschaftlichen Beirat begleitet und beraten.

Karger Kompass Onkol 2014;1:47

Tagungen und Kongresse

2014

FEBRUAR

31. Deutscher Krebskongress 2014

19.-22. Februar 2014

Berlin (Deutschland)

www.dkk2014.de/willkommen.html

Psychosoziale Onkologie - Fallbesprechung für Ärzte aller Fachgruppen 19. Februar 2014 und 24. September 2014 Hamburg (Deutschland) www.aekhh.de/

\section{German Breast Group (GBG) Jahrestreffen}

27.-28. Februar 2014

Frankfurt/M. (Deutschland)

www.germanbreastgroup.de/veranstaltungen. html?lang=de_DE.UTF-8\%2C+de_CH.U

\section{MÄRZ}

Frühjahrestagung der Deutschen Gesellschaft für Hämatologie und Onkologie (DGHO)

13.-14. März 2014

Berlin (Deutschland)

www.dgho-service.de/tagungen_seminare_ weiterbildungen/dgho_fruhjahrstagung

\section{European Lung Cancer Conference}

26.-29. März 2014

Genf (Schweiz)

www.esmo.org/Conferences/ELCC-2014-LungCancer

\section{AOI-GI-Tag: Arbeitstreffen der AIO-} Arbeitsgruppen mit dem Schwerpunkt Gastrointestinale Tumoren

\section{8.-29. März 2014}

Berlin (Deutschland)

www.aio-portal.de/index.php/termineveranstaltungen.html

\section{APRIL}

8. Gynäkologie-Onkologie-UpdateSeminar Gyn-Onko Update 2014 04.-05. April 2014

Wiesbaden (Deutschland) www.gyn-onko-update.com/
MAI

IMPAKT 2014 Breast Cancer Conference 08.-10. Mai 2014

Brüssel (Belgien)

www.esmo.org/Conferences/IMPAKT-2014Breast-Cancer

JUNI

10. Kongress der Deutschen Gesellschaft für Palliativmedizin und der 13. Deutsche Kongress für Versorgungsforschung

24.-27. Juni 2014

Düsseldorf (Deutschland)

www.dgp-dkvf2014.de/

JULI

20. Jahrestagung der Deutschen Gesellschaft für Radioonkologie DEGRO

03.-06. Juli 2014

Düsseldorf (Deutschland)

www.degro.org/degro2014/

GI-Oncology 2014 - 10. Interdisziplinäres Update der Arbeitsgemeinschaft für Internistische Onkologie

05. Juli 2014

Wiesbaden (Deutschland)

www.gi-oncology.de/

\section{SEPTEMBER}

Jahrestagung der European Society for Medical Oncology (ESMO) 2014

26.-30. September 2014

Madrid (Spanien)

www.esmo.org/events/madrid-2014-

esmo-congress.html

\section{OKTOBER}

Gemeinsame Jahrestagung der DGHO, ÖGHO, SGMO und SGH

10.-14. Oktober 2014

Hamburg (Deutschland) www.haematologie-onkologie-2014.com

\title{
KARGER
}

() 2014 S. Karger GmbH, Freiburg 